\title{
Correction to: Efficient Processing of Convolutional Neural Networks on SW26010
}

Yi Zhang, Bing Shu, Yan Yin, Yawei Zhou, Shaodi Li, and Junmin $\mathrm{Wu}$

\section{Correction to: \\ Chapter "Efficient Processing of Convolutional Neural \\ Networks on SW26010" in: X. Tang et al. (Eds.): \\ Network and Parallel Computing, LNCS 11783, https://doi.org/10.1007/978-3-030-30709-7_26}

In the originally published version of this chapter, in section 2.2 and 3.3, in the second to last sentence "swDGEMM" was corrected to "swDNN". Furthermore, in the last sentence of 3.3 , "16" was corrected to " 17 ", and a reference to https://github.com/ feifeibear/swDNNv1.0 has been added. 